\title{
Biodiversity of one of the Mishing villages of Assam with an emphasis of the traditional knowledge and use of the wild plants
}

\author{
Dutta Mary ${ }^{1}$, Basumatari Mallika ${ }^{1}$, Borah Darshana ${ }^{1}$, Gogoi Lina ${ }^{2}$ \\ ${ }^{1}$ Balipara Tract and Frontier Foundation, Sonitpur, Assam, India \\ ${ }^{2}$ Department of Energy, Tezpur University, Napaam, Tezpur, Assam, India \\ Corresponding Author: lina.dbr@gmail.com
}

\author{
A R T I C L E I N F O \\ Received: 09 June 2018 | Accepted: 04 October 2018 | Published Online: 31 December 2018 \\ DOI: $10.31786 / 09756272.18 .9 .2 .212$ \\ EOI: 10.11208/essence.18.9.2.212 \\ Article is an Open Access Publication. \\ This work is licensed under Attribution-Non Commercial 4.0 International \\ (https://creativecommons.org/licenses/by/4.0/) \\ CThe Authors (2018). Publishing Rights @ MANU_ICMANU \& ESSENCE_IJERC.
}

\section{A B S T R A C T}

The Mishing tribe is one of the hordes of the Mongoloid groups of people occupying the hills and valleys of northeastern India. Mishing or Miri tribe inhabiting the districts of Dhemaji, North Lakhimpur, Sonitpur, Tinsukia, Dibrugarh, Sibsagar, Jorhat and Golaghat of Assam, Northeast India. Many of the wild plants are used in the livelihood strategies of the tribal people. The present study highlights the biodiversity of one of the Mishing tribe villages of Assam with an emphasis on the traditional knowledge and use of these wild plants. A total of 31 plant and 19 bird species were recorded in the present study. In our survey, 67 species of butterflies of various families are reported in the village. Two species namely, Santalum album L. and Cycas pectinata are vulnerable and Swietenia mahagoni (L.) is endangered in the IUCN red list. Among the bird species, Leptoptilos javanicus is vulnerable in the IUCN red list.

\section{K E Y W O R D S}

Santalum album L | Cycas pectinate | Swietenia mahagoni (L.) | Leptoptilos javanicus | IUCN red list

\section{I T A T I O N}

Dutta, Mary; Basumatari, Mallika; Borah, Darshana and Gogoi, Lina (2018): Biodiversity of one of the Mishing villages of Assam with an emphasis of the traditional knowledge and use of the wild plants. ESSENCE Int. J. Env. Rehab. Conserv. IX (1): 80—86. 


\section{Introduction}

The Mishing tribe is one of the hordes of the Mongoloid groups of people occupying the hills and valleys of northeastern India. The Mishing tribal community belonged to Mongoloid group a multitude of people that followed Austro-Asiatic races to India (Singh et al., 1996). Mishing or Miri tribe inhabiting the districts of Dhemaji, North Lakhimpur, Sonitpur, Tinsukia, Dibrugarh, Sibsagar, Jorhat and Golaghat of Assam, Northeast India. Many of the wild plants are used in the livelihood strategies of the tribal people. A good number of wild plants are traditionally used by the Mishing people in their day to day life (Barua et al., 2007). The present study highlights the biodiversity of one of the Mishing tribe villages of Assam with an emphasis of the traditional knowledge and use of these wild plants

\section{Materials and Methods}

Study area: The study was conducted in the Baligaon Miri Green village of Sonitpur district of Assam, Northeast India. Sonitpur district is spread over on the northern banks of Brahmaputra, the lifeline of Assam. It falls in the Tropical Rainforest climate region that enjoys both hot and wet climatic condition. Summers are hot and humid with an average temperature of $27^{\circ} \mathrm{C}$.

Survey method: Surveys were carried out at different spots of the village band its vicinity by line transects method (Barhaum et al., 1980). Field notes, photographs and observations of the flora and fauna were taken for all the seasons during the daylight hours. Species were noted along with the time, date and location of capture. The flora and fauna of the village are critically surveyed in different localities for one complete year.

Taxonomic study: Identification of flora and fauna was done with the help of literature. Digital photographs were taken for both flora and fauna identified.

\section{Discussion}

A total of 31 plant species belonging to 27 families and 19 bird species belonging to 18 families were recorded in the present study. In our survey, 67 species of butterflies of various families are reported in the campus and out of 6 families 5 different families are present in the study area. Out of 31 plant species, 2 species namely, Santalum album L. and Cycas pectinata are vulnerable and Swietenia mahagoni (L.) is endangered in the IUCN red list. Among the bird species, Leptoptilos javanicus is vulnerable in the IUCN red list. Most of the plants have traditional medicinal value and many are used as food in the Mishing communities (listed in table 1). Most of Mishing people cultivated mainly four types of rice: Aijong, Bora, Bao, and Ranjit. Apart from these, they cultivated almost all type of vegetable grown in this geographical region and used to sell in the local market. Leaves of Clerodendron serratum (L.) Moon also used in the preparation of Apong which has a significant role in Mishing culture and tradition. The Mishing tribe is one of the hordes of the Mongoloid groups of people occupying the hills and valleys of northeastern India. Mishing or Miri tribe inhabiting the districts of Dhemaji, North Lakhimpur, Sonitpur, Tinsukia, Dibrugarh, Sibsagar, Jorhat and Golaghat of Assam, Northeast India. Many of the wild plants are used in the livelihood strategies of the tribal people. The present study highlights the biodiversity of one of the Mishing tribe villages of Assam with an emphasis on the traditional knowledge and use of these wild plants. A total of 31 plant and 19 bird species were recorded in the present study. In our survey, 67 species of butterflies of various families are reported in the village. Two species namely, Santalum album L. and Cycas pectinata are vulnerable and Swietenia mahagoni (L.) is endangered in the IUCN red list. Among the bird species, Leptoptilos javanicus is vulnerable in the IUCN red list. 


\section{Observations}

\begin{tabular}{|c|c|c|c|c|}
\hline S. No. & Scientific name & English name & Family & Plant parts used \\
\hline 1. & Vicia benghalens L. & Purple vetch & Faboideae & \\
\hline 2. & Ficus hispid L.f. & Indian Ivy & Moraceae & \\
\hline 3. & Streblus asper Lour. & $\begin{array}{l}\text { Siamese rough } \\
\text { bush }\end{array}$ & Moraceae & Medicinal value \\
\hline 4. & $\begin{array}{l}\text { Sarcochlamys pulcherrima } \\
\text { Gaud. }\end{array}$ & Duggal fibre tree & Urticaceae & Edible, Young leaves \\
\hline 5. & $\begin{array}{l}\text { Benincasa hispida (Thunb. ex } \\
\text { Murray) Cogn. }\end{array}$ & Wax gourd & Cucurbitaceae & Fruit \\
\hline 6. & $\begin{array}{l}\text { Colocasia esculenta (L.) } \\
\text { Schott }\end{array}$ & Cocoyam & Araceae & $\begin{array}{l}\text { Tender leaves, tubers } \\
\text { (Mishing edible) }\end{array}$ \\
\hline 7. & Amaranthus caudatus L. & Love-lies-bleeding & Amaranthaceae & $\begin{array}{l}\text { Leaves and seeds } \\
\text { Edible }\end{array}$ \\
\hline 8. & Manihot esculenta & Cassava & Euphorbiaceae & Roots edible \\
\hline 9. & Terminalia arjuna (Roxb.) & White murdah & Combretaceae & \\
\hline 10. & Costus speciosus & $\begin{array}{l}\text { Elegant Costus, } \\
\text { Kusta }\end{array}$ & Costaceae & $\begin{array}{l}\text { Medicinal, ornamental } \\
\text { value }\end{array}$ \\
\hline 11. & $\begin{array}{l}\text { Flacourita indica (Burm. f.) } \\
\text { Merr. }\end{array}$ & Governor's Plum & Flacourtiaceae & $\begin{array}{l}\text { Medicinal value, Use in } \\
\text { snake bite also }\end{array}$ \\
\hline 12. & $\begin{array}{l}\text { Santalum album L. IUCN } \\
\text { status: VU }\end{array}$ & Sandal Wood & Santalaceae & \\
\hline 13. & Tinospora cordifolia & Gulanch, Guduchi & Menispermaceae & Medicinal value \\
\hline 14. & Stemonia tuberose Lour. & & Stemonaceae & Medicinal value \\
\hline 15. & Spilanthes acmella Murr. & Pellitary & Compositae & Medicinal value \\
\hline 16. & Hibiscus cannabinus L. & Kenaf & Malvaceae & Edible \\
\hline 17. & $\begin{array}{l}\text { Clerodendron serratum (L.) } \\
\text { Moon }\end{array}$ & $\begin{array}{l}\text { Blue glory, Beetle } \\
\text { killer }\end{array}$ & Lamiaceae & Medicinal value \\
\hline 18. & Ananas comosus (L.) Merr. & Pineapple & Bromeliaceae & Fruit \\
\hline 19. & Abelmoschus moschatus & Devil's Cotton & Malvaceae & Medicinal value \\
\hline 20. & Centella asiatica (L.) Urb. & Indian pennywort & Apiaceae & $\begin{array}{l}\text { Edible: whole plant, } \\
\text { Medicinal value }\end{array}$ \\
\hline 21. & $\begin{array}{l}\text { Artocarpus heterophyllus } \\
\text { Lamk. }\end{array}$ & Jackfruit tree & Moraceae & Edible \\
\hline 22. & Calamus rotang $\mathrm{L}$. & Rattang palm, cane & Arecaceae & \\
\hline 23. & Citrus paradise & Lemon & Rutaceae & Fruit \\
\hline 24. & Tinospora crispa & $\begin{array}{l}\text { Patawali, } \quad \text { Akar } \\
\text { Patawali }\end{array}$ & Menispermaceae & Medi cinal value \\
\hline 25. & $\begin{array}{l}\text { Cycas pectinata IUCN } \\
\text { status: VU }\end{array}$ & Sago Palm & Cycadaceae & \\
\hline 26. & Phoebe hainesiana & Bonsum & Lauraceae & \\
\hline 27. & Tectona grandis & Teak & Lamiaceae & Timber \\
\hline 28. & $\begin{array}{l}\text { Swietenia mahagoni (L.) IUCN } \\
\text { status: EN }\end{array}$ & Mahogany wood & Meliaceae & \\
\hline 29. & Chukrasia tabularis A. Juss. & Bastard cedar & Meliaceae & \\
\hline 30. & Dalbergia sissoo & Indian rosewood & Fabaceae & Medicianl value \\
\hline 31. & Shorea robusta & Sal tree & Dipterocarpaceae & $\begin{array}{l}\text { Timber, Medicinal } \\
\text { value }\end{array}$ \\
\hline 32. & Psidium guajava L. & Guava & Myrtaceae & Friut \\
\hline 33. & Magnifera indica L. & Mango & Anacardiaceae & Fruit \\
\hline
\end{tabular}

Table 1. Plant species found in Baligaon, Assam 
ESSENCE—IJERC | Mary et.al., (2018) | IX (2): 80-86

\begin{tabular}{|c|c|c|c|}
\hline S. No. & Scientific name & $\begin{array}{l}\text { Common name/ English } \\
\text { name }\end{array}$ & Family \\
\hline 1. & Phalacrocorax niger & Little Cormorant & Phalacrocoracidae \\
\hline 2 & Acridotheres tristis & Common Myna & Sturnidae \\
\hline 3. & Streptopelia chinensis & Spotted dove & Columbidae \\
\hline 4. & Pycnonotus cafer & Red-vented bulbul & Pycnonotidae \\
\hline 5. & Pteropus vampyrus & Large flying fox & Pteropodidae \\
\hline 6. & Passer domesticus & House sparrow & Passeridae \\
\hline 7. & Aethopyga siparaja & Crimson sunbird & Nectariniidae \\
\hline 8. & Copsychus saularis & Oriental Magpie robin & Muscicapidae \\
\hline 9. & Dendrocygna javanica & lesser whistling duck & Anatidae \\
\hline 10. & Ardea alba & Large Egret & Ardeidae \\
\hline 11. & Amauronis phoenicrus & The white-breasted waterhen & Rallidae \\
\hline 12. & Corvus splendens & House crow & Corvidae \\
\hline 13. & Leptoptilos javanicus IUCN Status: VU & Lesser Adjutant & Ciconiidae \\
\hline 14. & Vanellus indicus & Redwattled lapwing & Charadriidae \\
\hline 15. & Dicrurus macrocercus & Black Drongo & Dicruridae \\
\hline 16. & Dendrocitta vagabunda & Rufous Treepie & Corvidae \\
\hline 17. & Lanius cristatus & Brown Shrike & Laniidae \\
\hline 18. & Coracies benghalensis & Indian Roller & Coraciidae \\
\hline 19. & Halcyon smyrnensis & White - throated Kingfisher & Alcedinidae \\
\hline
\end{tabular}

Table 2: Bird species found in Baligaon, Assam

\begin{tabular}{|l|l|l|l|}
\hline S. No. & Scientific name & Common name & Family \\
\hline 1. & Celaenorrhinus leucocera & Common Spotted Flat & Hesperiidae \\
\hline 2. & Telicota ancilla & Dark Palm Dart & Hesperiidae \\
\hline 3. & Odontoptilum angulate & Chestnut Angle & Hesperiidae \\
\hline 4. & Notocrypta curvifascia & Restricted Demon & Hesperiidae \\
\hline 5. & Spialia galba & Indian Skipper & Hesperiidae \\
\hline 6. & Pseudocoladenia dan & Fulvous Pied Flat & Hesperiidae \\
\hline 7. & Udaspes folus & Grass Demon & Hesperiidae \\
\hline 8. & Telicota colon & Pale Palm Dart & Hesperiidae \\
\hline 9. & Iambrix salsala & Chestnut Bob & Hesperiidae \\
\hline 10. & Taractrocera maevius & Common Grass Dart & Hesperiidae \\
\hline 11. & Sarangesa dasahara & Common Small Flat & Hesperiidae \\
\hline 12. & Common Dartlet & Oriens gola & Hesperiidae \\
\hline 13. & Troides Helena & Common Birdwing & Papilionidae \\
\hline 14. & Papilio Helenus & Red Helen & Papilionidae \\
\hline 15. & Graphium sarpedon & Common Bluebottle & Papilionidae \\
\hline 16. & Papilio iswara & Papilionidae \\
\hline
\end{tabular}


ESSENCE—IJERC | Mary et.al., (2018) | IX (2): 80-86

\begin{tabular}{|c|c|c|c|}
\hline 17. & Papilio polytes & Common Mormon & Papilionidae \\
\hline 18. & Papilio Memnon & Great Mormon & Papilionidae \\
\hline 19. & Papilio demoleus & Lime Butterfly & Papilionidae \\
\hline 20. & Graphium doson & Common Jay & Papilionidae \\
\hline 21. & Graphium Agamemnon & Tailed Jay & Papilionidae \\
\hline 22. & Papilio clytia & Common Mime & Papilionidae \\
\hline 23. & Pachliopta aristolochiae & Common Rose & Papilionidae \\
\hline 24. & Catopsilia Pomona & Common Emigrant & Pieridae \\
\hline 25. & Catopsilia pyranthe & Mottled Emigrant & Pieridae \\
\hline 26. & Eurema sari & Chocolate grass yellow & Pieridae \\
\hline 27. & Pieris brassicae & Large Cabbage White & Pieridae \\
\hline 28. & Eurema brigitta & Small grass Yellow & Pieridae \\
\hline 29. & Pieris canidia & Indian Cabbage White & Pieridae \\
\hline 30. & Cepora nadina & Lesser Gull (Rare) & Pieridae \\
\hline 31. & Cepora Nerissa & Common Gull & Pieridae \\
\hline 32. & Colotis aurora & Plain Orange Tip & Pieridae \\
\hline 33. & Ixias Marianne & White Orange Tip & Pieridae \\
\hline 34. & Hebomoia glaucippe & Great Orange Tip & Pieridae \\
\hline 35. & Colotis etrida & Small orange tip & Pieridae \\
\hline 36. & Delias eucharis & Common Jezebel & Pieridae \\
\hline 37. & Eurema hecabe & Common Grass Yellow & Pieridae \\
\hline 38. & Eurema hecabe & Grass yellow sp. & Pieridae \\
\hline 39. & Junonia iphita & Chocolate Pansy & Nymphalidae \\
\hline 40. & Junonia lemonias & Lemon Pansy & Nymphalidae \\
\hline 41. & Junonia almanac & Peacock Pansy & Nymphalidae \\
\hline 42. & Junonia hierta & Yellow Pansy & Nymphalidae \\
\hline 43. & Junonia atlites & Gray Pancy & Nymphalidae \\
\hline 44. & Phalanta phalantha & Common Leopard & Nymphalidae \\
\hline 45. & Cethosia biblis & Red Lacewing & Nymphalidae \\
\hline 46. & Danaus genutia & Common Tiger & Nymphalidae \\
\hline 47. & Cethosia cyane & Leopard Lacewing & Nymphalidae \\
\hline 48. & Ypthima huebneri & Common Four Ring & Nymphalidae \\
\hline 49. & Orsotriaena medus & Nigger & Nymphalidae \\
\hline 50. & Ypthima baldus & Common Five Ring & Nymphalidae \\
\hline 51. & Ariadne Ariadne & Angled Castor & Nymphalidae \\
\hline 52. & Melanitis leda & Common Evening Brown & Nymphalidae \\
\hline 53. & Mycalesis perseus & Common Bush Brown & Nymphalidae \\
\hline 54. & Neptis hylas & Common Sailer & Nymphalidae \\
\hline 55. & Athyma nefte & Colour Sergeant & Nymphalidae \\
\hline
\end{tabular}


ESSENCE—IJERC | Mary et.al., (2018) | IX (2): 80-86

\begin{tabular}{|l|l|l|l|}
\hline 56. & Athyma perius & Common Sergeant & Nymphalidae \\
\hline 57. & Parantica aglea & Glassy Tiger & Nymphalidae \\
\hline 58. & Moduza procris & Commander & Nymphalidae \\
\hline 59. & Euploea core & Common Indian Crow & Nymphalidae \\
\hline 60. & Tanaecia lepidea & Grey Count & Nymphalidae \\
\hline 61. & Chilades lajus & Lime Blue & Lycaenidae \\
\hline 62. & Jamides celeno & Common Cerulan & Lycaenidae \\
\hline 63. & Castalius rosimon & Common Pierrot & Lycaenidae \\
\hline 64. & Cheritra freja & Common Imperial & Lycaenidae \\
\hline 65. & Loxura atymnus & Yamfly & Lycaenidae \\
\hline 66. & Zizula hylax & Tiny Grass Blue & Lycaenidae \\
\hline 67. & Curetis thetis & Indian Sunbeam & Lycaenidae \\
\hline
\end{tabular}

Table 3. Butterfly species found in Baligaon, Assam

\begin{tabular}{|l|l|l|l|}
\hline S. No. & Scientific name & Common name & Family \\
\hline 1. & Argiope aurantia & Yellow garden spider & Araneidae \\
\hline 2. & Eriophora transmarina & Garden Orbweaver & Araneidae \\
\hline 3. & Palystes castaneus & Huntsman spider & Sparassidae \\
\hline 4. & Oxyopes salticus & Lynx spider & Oxyopidae \\
\hline 5. & Nephila clavipes & Golden orb-weaver & Araneidae \\
\hline 6. & Deilephila elpenor & Hawk moth caterpiller & Lepidoptera \\
\hline 7. & Lithobius forficatus & Centepede & Lithobiidae \\
\hline 8. & Calotes versicolor & Oriental garden lizard & Agamidae \\
\hline 9. & Ancistrocerus sp. & Potter wasp & Vespidae \\
\hline
\end{tabular}

Table 4. Insects found in Baligaon, Assam

\begin{tabular}{|l|l|l|l|}
\hline S. No. & Scientific name & Common name & Family \\
\hline 1. & Lycodon flavomaculatus & Yellow speckled wolf snake & Colubridae \\
\hline 2. & Lycodon capucinus & Common wolf snake & Colubridae \\
\hline 3. & Coelognathus radiatus & Juvenile Copper headed trinket & Colubridae \\
\hline 4. & Dendrelaphis pictus & Painted bronzeback & Colubridae \\
\hline 5. & Dendrelaphis cyanochloris & Blue bronzeback & Colubridae \\
\hline 6. & Amphiesma stolatum & Striped keelback & Colubridae \\
\hline 7. & Xenochrophis piscator & Checkered Keelback & Colubridae \\
\hline 8. & Naja naja & Spectacled cobra & Elapidae \\
\hline 9. & Boiga quincunciata & Assamese Cat Snake & Colubridae \\
\hline 10. & Trimeresurus albolabris & White lipped pit viper & Viperidae \\
\hline 11. & Gekko gecko & Tokay Gecko & Gekkonidae \\
\hline
\end{tabular}

Table 5. Reptiles found in Baligaon, Assam 
ESSENCE—IJERC | Mary et.al., (2018) | IX (2): 80—86

\begin{tabular}{|l|l|l|l|}
\hline S. No. & Scientific name & Common name & Family \\
\hline 1. & Duttaphrynus melanostictus & Common Asian toad & Bufonidae \\
\hline 2. & Acris crepitans & Cricket frog & Hylidae \\
\hline 3. & Rana leptoglossa & Hylarana leptoglossa & Ranidae \\
\hline 4. & Microhyla ornata & Microhyla ornate & Microhylidae \\
\hline
\end{tabular}

Table 6. Amphibians found in Baligaon, Assam

\section{Acknowledgement}

The authors would like to offer their sincere thanks to Balipara Tract and Frontier Foundation and Wild Mahseer. The first authors gratefully acknowledge the financial grant from Globally Managed Services (GMS) and Tata Trust.

\section{Reference}

Barhaum, K. P.; Anderson, D. R. and Cauke, Z. L. (1980): Estimation of density from line transects sampling of biological population. Wildlife Monographs. 72, 515.
Barua, U.; Hore, D. K. and Sarma, R. (2007): Wild edible plants of Majuli island and Darang districts of Assam. Indian Journal of Traditional Knowledge. 6, 191-194.

Singh, J.; Bhuyan, T. C. and Ahmed, A. (1996): Ethnobotanical studies on the Mishing tribes of Assam with special reference to food and medicinal plant. Journal Economic Taxonomy Botany. 12, 350356. 